\title{
Efectos sobre microdureza y grado de conversión de dos tipos de resinas sometidas a tratamientos de pospolimerización
}

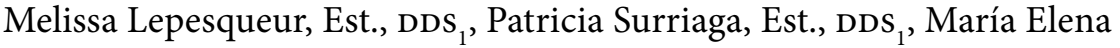 \\ Masache, Est., DDs 1 , Berenice Vásquez, Est., DDs ${ }_{1}$, Mauricio Peña ${ }_{1}$, Osnara \\ María Mongruel Gomes, PhD. ${ }_{2}$, John Alexis Domínguez ${ }^{\star}, \mathrm{PhD}_{{ }_{1,2}}$
}

Departamento de Rehabilitación Oral, Universidad Hispano-Guarani, Asunción, Paraguay ${ }_{2}$ Universidad Estadual de Ponta Grossa, Ponta Grossa, Brasil

Recibido: 14 de mayo del 2015. Aprobado: 19 de junio del 2015.

*Autor de correspondencia: John Alexis Domínguez. Universidad Estadual de Ponta Grossa, Ponta Grossa, Brasil. Alameda Nabuco de Araujo 422, Barrio Uvaranas, cep: 84031510. Teléfono 554298280372. Correo electrónico: johnalexis. dominguez@gmail.com.

Cómo citar este artículo: Lepesqueur M, Surriaga P, Masache ME, Vásquez B, Peña M, Gomes OMM, Domínguez JA. Efectos sobre microdureza y grado de conversión de dos tipos de resinas sometidas a tratamientos de pospolimerización. Rev Nac Odontol. 2015;11(21):49-56. doi: http://dx.doi.org/10.16925/od.v11i21.947

Resumen. Objetivo: evaluar la microdureza y el grado de conversión después de diferentes técnicas poscurado en dos tipos de resinas compuestas. Materiales y métodos: se realizaron 36 cuerpos de prueba con $12 \mathrm{~mm}$ de diámetro y $4 \mathrm{~mm}$ de altura, con dos resinas: Filtek z350 y Ena Miscerium. Después de la confección, los cuerpos de prueba se llevaron al microduremetro y a espectroscopia de infrarrojo, y fueron sometidos a cuatro procesos de pospolimerización: agua en ebullición, microonda, calor seco, autoclave y horno gradia. Resultados: se hizo análisis estadístico por medio de Anova de dos factores con postest Tukey por resina evaluada, se presentan media y desviación estándar, los valores de grado de conversión muestran un gran aumento en la resina Ena comparado con el grupo control (63,81\%), cuando es realizado pospolimerización con calor seco $(89,02 \%)$ y una gran disminución cuando es realizado con agua en ebullición $(36,41 \%)$. Conclusiones: el proceso de curado posterior por microondas y autoclave aumenta los valores de microdureza y grado de conversión, y el agua destilada a temperaturas altas disminuye la microdureza de la superficie de las resinas compuestas.

Palabras clave: grado de conversión, microdureza, resinas compuestas. 


\title{
Effects on Micro-Hardness and Conversion Level of Two Types of Resins Undergoing Post-Polymerization Treatments
}

\begin{abstract}
Objetive: to evaluate micro-hardness and conversion levels after different postcuring techniques in two types of composite resins. Materials and methods: $3612 \mathrm{~mm}$ diameter $4 \mathrm{~mm}$-height specimens were manufactured with two resins: Flitek z350 and Ena Miscerium. After the manufacturing, specimens were taken to the microduremeter and infrared spectroscopy, and endured four post-polymerization processes: boiling water, microwave, dry heat, autoclave and horno gradia. Results: statistical analyses (by Anova) of two factors with Tukey's Post Hoc Test by evaluated resin was performed, there appear media and standard deviation, conversion level degrees show a large increase in Ena resin compared to the control group $(63.81 \%)$ when dry heat post-polymerization is performed (89.02\%) and a large decrease when it is performed with boiling water (36.41\%). Conclusions: the post-curing process by microwave and autoclave increase micro-hardness level and conversion level, while distilled water at high temperatures diminishes superficial micro-hardness of composite resins.
\end{abstract}

Keywords: degree of conversion, micro-hardness, composite resins.

\section{Efeitos sobre microdureza e grau de conversão de dois tipos de resinas submetidas a tratamentos de pós-polimerização}

Resumo. Objetivo: avaliar a microdureza e o grau de conversão depois de diferentes técnicas pós-curado em dois tipos de resinas compostas. Materiais e métodos: realizaram-se 36 corpos de teste com $12 \mathrm{~mm}$ de diâmetros e $4 \mathrm{~mm}$ de altura, com duas resinas: Filtek z350 eEna Miscerium. Depois da confecção os corpos de teste foram levados ao microdurômetro e a espectroscopia de infravermelho, e submetidos a quatro processos de pós-polimerização: água em ebulição, micro-onda, calor seco, autoclave e Horno Gradia. Resultados: realizouse análise estatística mediante de Anova de dois fatores com pós-teste de Tukey por resina avaliada, apresentam-se média e desvio padrão, os valores de grau de conversão evidenciam um grande aumento na resina Ena comparado com o grupo controle $(63,81 \%)$, quando realizado pós-polimerização com calor seco $(89,02 \%)$ e uma grande diminuição quando realizado com água em ebulição (36,41\%). Conclusões: o processo de curado posterior por micro-ondas e autoclave aumenta os valores de microdureza e grau de conversão, e a água destilada a temperaturas altas diminui a microdureza da superfície das resinas compostas.

Palavras-chave: grau de conversão, microdureza, resinas compostas. 


\section{Introducción}

Desde la aparición de las resinas compuestas (1960) [1], los avances han sido muchos con respecto a su composición y utilización; sin embargo, las restauraciones de resinas compuestas presentan deficiencias en el sellado marginal como consecuencia de la contracción de polimerización [2]. Para compensar estos inconvenientes, los sistemas de resinas compuestas indirectas representan la mejor alternativa.

La primera generación de resinas compuestas indirectas para posteriores en la década de 1980 [3] mostró un mejor desempeño mecánico, permitiendo elevar el grado de conversión del material restaurador mediante proceso adicional de poscurado y la reducción significativa de la contracción de polimerización [4]; beneficios clínicos adicionales incluyen adaptación marginal, ideales contactos proximales, excelente morfología y estética óptima [5].

Las restauraciones de resina compuesta indirecta han demostrado menor microfiltración en la interfase diente-restauración, debido a la eliminación del estrés contracción y contracción volumétrica [6]. A pesar de esta disminución, se han encontrado monómeros libres no polimerizados que indican la necesidad de complementar uniones de dupla carbono por medio de procesos alternativos de curado [7]. En estudios anteriores, autores como Sharafeddin et al. [8] probaron la eficacia de los métodos alternativos complementarios para poscurado en resinas directas y concluyeron que el autoclave, microondas u horno eran eficientes en dicho proceso, aumentando la dureza superficial del material.

Por estas razones, el objetivo del equipo de trabajo es evaluar la microdureza y el grado de conversión después de diferentes técnicas poscurado en dos tipos de resinas compuestas.

\section{Materiales y métodos}

Se utilizaron dos tipos de resina compuesta, una de nanorelleno Filtek ${ }^{\mathrm{mm}}$ Z350XT (3M ESPE) y otra nanohíbrida Enamel Plus HRI (Micerium; tabla 1).

Tabla 1. Resinas utilizadas, composición y casa comercial

\begin{tabular}{|c|c|c|}
\hline Material & Composición & Casa comercial \\
\hline Filtek $^{\mathrm{TN}} \mathrm{Z} 350 \mathrm{XT}$ (figura 1) & $\begin{array}{l}\text { BisGMA, TEGDMA y Procrylat. Relleno de trifluoruro de iterbio, un } \\
\text { relleno de sílice y un relleno de aglomeración de sílice/zirconia. El } \\
\text { agregado tiene un tamaño de partículas de clúster promedio de } 0,6 \text { a } \\
10 \text { micrones. La carga de relleno inorgánico es de aproximadamente } \\
46 \% \text { del volumen. }\end{array}$ & $3 M$ ESPE \\
\hline Enamel Plus HRI (figura 1) & $\begin{array}{l}\text { Matriz resinosa: diuretandimetacrilato; } 1,4 \text { Butandioldimetacrila- } \\
\text { to. Contenido del relleno: } 77 \% \text { en peso. Relleno vítreo: tamaño de } \\
\text { partículas medio } 4,3 \text { micron y } 0,7 \text { micron. Bióxido de silicio a alta } \\
\text { dispersión: dimensión media de las partículas } 0,04 \text { micron. }\end{array}$ & Micerium \\
\hline Lámpara de fotocurado Bluephase (G2) & & ivoclar vivadent LED \\
\hline
\end{tabular}

Fuente: elaboración propia

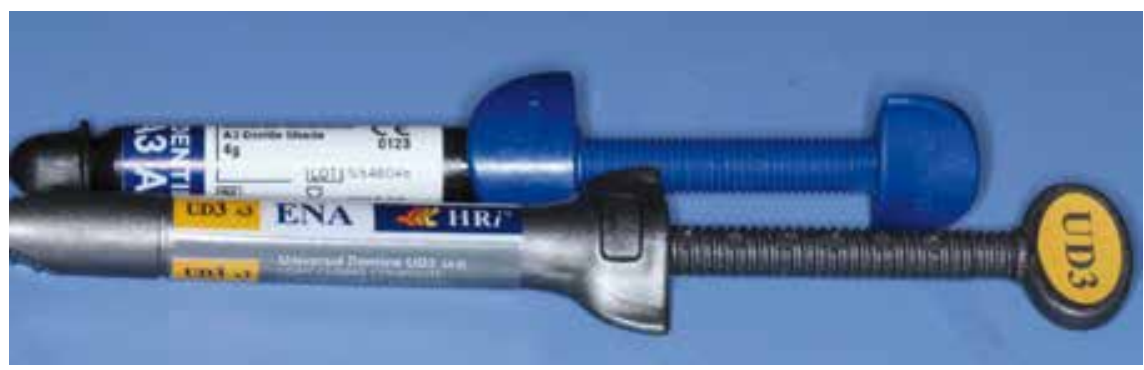

Figura 1. Resinas utilizadas en el estudio. Superior: Filtek Z350 XT, inferior: Enamel plus HRI Fuente: elaboración propia 


\section{Elaboración especímenes de prueba}

En el diseño de los dispositivos de prueba, se utilizó un dispositivo para confeccion de los cuerpos de prueba de acero inoxidable (figura 2A) con $12 \mathrm{~mm}$ de diámetro y $4 \mathrm{~mm}$ de altura. Se utiliza una loseta de vidrio como base, encima de la cual se interpone una tira de mylar con el fin de obtener una superficie homogénea de la resina Enamel Plus HRI (figura 2B); se coloca la matriz de acero encima de la tira de mylar y sobre la loseta, se procede a condensar la resina con un incremento de $2 \mathrm{~mm}$ estandarizados con sonda (Hu-Friedy; figura 2C). Este incremento se fotoactiva por 40 segundos y después de esto se hace un segundo incremento hasta obtener la altura de la matriz en acero; se interpone nuevamente una tira de mylar para dar homogeneidad a la superficie y se hace presión con otra loseta de vidrio para eliminar excesos y burbujas (figura 2D), se retira la loseta y se fotopolimeriza a $1 \mathrm{~mm}$ de distancia por 40 segundos más (figura 2E).

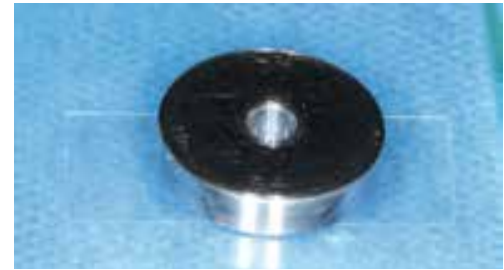

Figura 2A

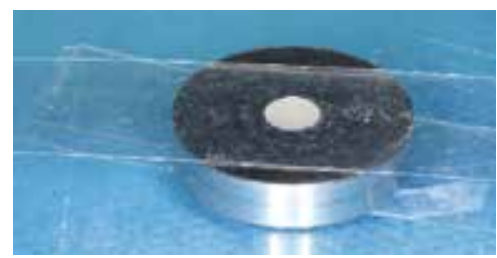

Figura 2D

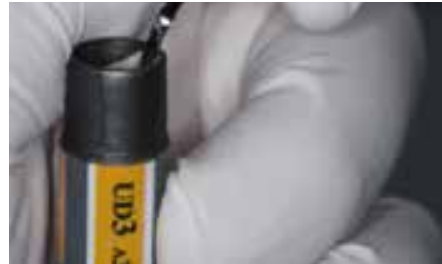

Figura 2B

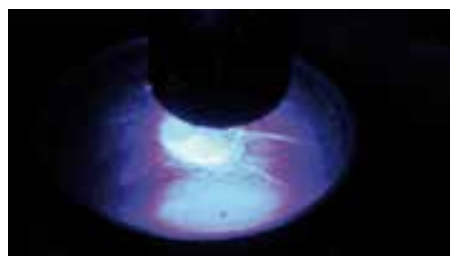

Figura 2E

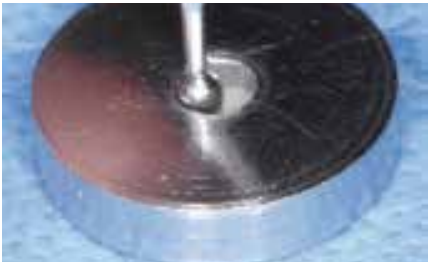

Figura 2C

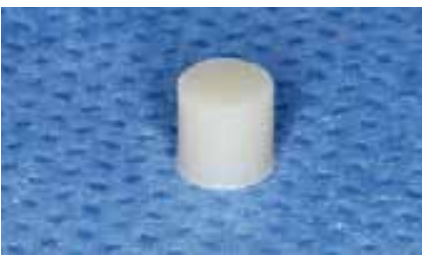

Figura 2F

Figura 2. 2A. Dispositivo para confección de cuerpos de prueba. 2B. Resina Ena. 2C. Colocación de resina en matriz. 2D. Matriz con resina incorporada para fotopolimerizacion. 2E. Fotopolimerizacion de resina en matriz. 2F. Cuerpo de prueba de resina Fuente: elaboración propia

Se elaboraron 36 discos de prueba para la resina compuesta Filtek $^{\text {tm }}$ Z350XT (3M ESPE) y 36 discos de prueba para Enamel Plus HRI (Micerium)
(Figura 2F), y se agruparon como se muestra en la figura 3.

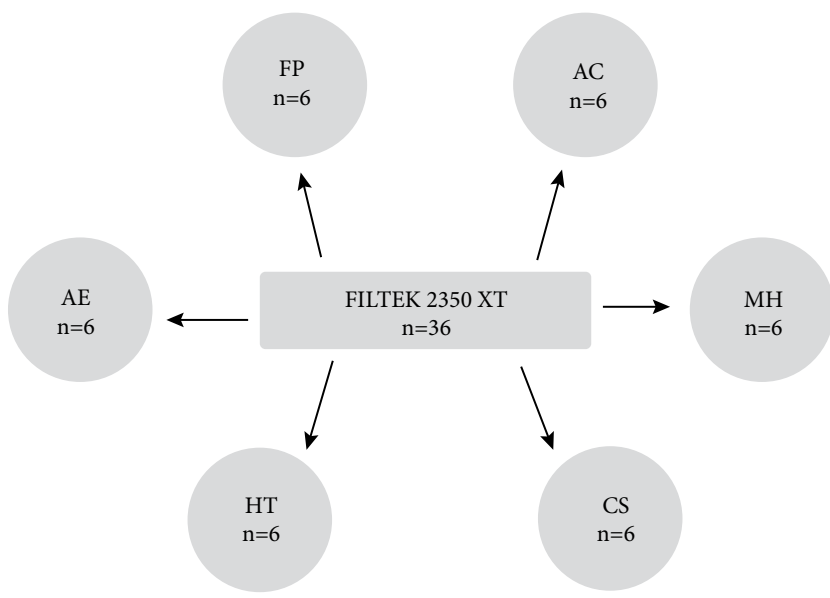

Figura 3. Distribución de cuerpos de prueba. $\mathrm{FP}=$ fotopolimerización. $\mathrm{AC}=$ Autoclave. $\mathrm{MH}=$ Microondas. $\mathrm{Cs}=\mathrm{Calor}$ seco. $\mathrm{HT}=$ Horno gradia. AE = Agua en ebullición. De igual forma se hizo para la resina ENA HRA Miscerium. 
Los discos de prueba se fotopolimerizaron durante 40 segundos cada incremento, con dos incrementos en su totalidad. Las técnicas de pos-fotopolimerización que se utilizaron para cada grupo se describen a continuación:

- Grupo control FP: 12 discos (divididos en 2 grupos de 6 correspondientes a cada material del compuesto) a los cuales se les realizó el procedimiento de fotopolimerización por 40 segundos.

- Grupo Ac: 12 discos (6 discos de cada material de prueba) después de fotopolimerizados fueron llevados a un autoclave 15 minutos en esterilización y 20 minutos de secado a una presión de 30 psi (unidad de presión equivalente a una libra por pulgada cuadrada), que corresponde a un ciclo normal de esterilización del instrumental según las recomendaciones del fabricante.

- Grupo AE: 12 discos (6 discos de cada material de prueba) después de fotopolimerizados se sumergieron en agua destilada en ebullición en una refractaria de vidrio templado, sobre una estufa convencional de gas natural durante 10 minutos a una temperatura de $95^{\circ} \mathrm{C}$ controlada con un termómetro de mercurio de laboratorio marca Brand, escala $-10^{\circ} \mathrm{C}$ hasta $250^{\circ} \mathrm{C}$, el cual se encontraba posicionado sobre un aditamento de acrílico.

- Grupo Hт: 12 discos (6 discos de cada material) se llevan a un horno Gradia (GC, Japan). Las muestras se posicionan dentro del horno durante 5 minutos por cada casa comercial.

- Grupo cs: 12 discos (6 discos de cada material de prueba) se llevan a un horno de calor seco (Biodent) durante 10 minutos a $125^{\circ} \mathrm{C}$.

- Grupo мн: 12 discos (6 discos de cada material) se llevan a un horno microondas (LG, China) durante 5 minutos en $200 \mathrm{ml}$ de agua destilada a una potencia de $480 \mathrm{~W}$.

Después de realizados todos los procedimientos de los grupos experimentales, se procede a hacer los test de microdureza y grado de conversión.

\section{Microdureza}

Los cilindros de resina fueron llevados al Laboratorio de Posgraduación de Odontología de la Universidad Estatal de Ponta Grossa, donde fue llevado a cabo el test de microdureza Knoop en el microdurómetro (Shimadzu Corporation, Kyoto, Japan), a una carga de 25 g y una velocidad de 15 segundos. En cada cilindro se realizaron 5 indentaciones en la base del cilindro.

\section{Grado de conversión}

Los cilindros fueron examinados en espectroscopia de infrarrojo para evaluar el grado de conversión (GC) en cada uno de los grupos de trabajo. Después del procedimiento realizado, se trituró un poco de la resina a evaluar $( \pm 1 \mathrm{mg})$ del tope del cuerpo de prueba y se mezcló con $100 \mathrm{mg}$ de brometo de potasio, para comprimir en una prensa hidráulica (Modelo ssp-10 ${ }^{\text {a }}$ ) con carga de $80 \mathrm{kN}$ durante 3 minutos y obtener así un disco de prueba. Los espectros de absorbancia se elaboraron analizando la región 400 hasta $4000 \mathrm{~cm} \mathrm{-1,}$ con la siguiente especificación: 64 escaneadas a una resolución de $4 \mathrm{~cm}-1$, y el grado de conversión fue calculado por:

$$
\begin{gathered}
\mathrm{GC}=\text { no polimerizado }\left(1607^{\star} 1637\right)^{\star} 100 \\
\text { polimerizado }\left(1607^{\star} 1637\right)
\end{gathered}
$$

\section{Resultados}

Después de hacer los análisis estadísticos de los datos obtenidos de microdureza, previa prueba de D’Agostino y Anova - dos factores con postest Tukey por resina evaluada-, en la tabla 2 se presentan la media, la desviación estándar y los porcentajes del grado de conversión de cada grupo experimental.

Los valores de grado de conversión muestran un gran aumento en la resina Ena comparado con el grupo control $(63,81 \%)$ cuando se realiza pospolimerización con calor seco $(89,02 \%)$, y una gran disminución cuando se realiza en agua con ebullición $(36,41 \%)$. En la resina Z350 no se presentó ninguna diferencia sobre el grado de conversión, pero en microdureza aumentó significativamente cuando fue hecha la pospolimerización con microonda (102 Knoop) comparado con el grupo control (61 Knoop) y de igual forma disminuyó con agua en ebullición (35 Knoop). 
Tabla 2. Porcentaje de grado de conversión dos resinas y 6 grupos pospolimerización, y media y desviación estandar de microdureza Knoop

\begin{tabular}{|l|c|c|c|c|}
\hline \multirow{2}{*}{} & \multicolumn{2}{|c|}{ Miscerium } & \multicolumn{2}{c|}{ Z 350 } \\
\cline { 2 - 5 } & $\begin{array}{c}\text { Grado de } \\
\text { conversión (\%) }\end{array}$ & Microdureza & $\begin{array}{c}\text { Grado de } \\
\text { conversión (\%) }\end{array}$ & Microdureza \\
\hline Control & 63,81 & $35,7 \pm 18,2 \mathrm{~A}$ & 74,36 & $61,2 \pm 17,3 \mathrm{~A}$ \\
\hline Agua & 36,41 & $40,0 \pm 18,2 \mathrm{~A}$ & 56,62 & $35,1 \pm 16,8 \mathrm{~B}$ \\
\hline Microonda & 51,93 & $70,7 \pm 12,3 \mathrm{~B}$ & 75,83 & $102 \pm 14,2 \mathrm{~B}$ \\
\hline Calor seco & 89,02 & $68,2 \pm 17,2 \mathrm{~B}$ & 54,01 & $55,5 \pm 21,3 \mathrm{~A}$ \\
\hline Autoclave & 51,24 & $79,8 \pm 19,4 \mathrm{~B}$ & 47,65 & $64,5 \pm 20,1 \mathrm{~A}$ \\
\hline Gradia & 52,44 & $45,2 \pm 16,2 \mathrm{~A}$ & 60,88 & $47,7 \pm 13,2 \mathrm{~A}$ \\
\hline${ }^{*}$ Letras diferentes denotan diferencia significativa de forma horizontal. \\
\hline \multicolumn{5}{|c}{} \\
\hline
\end{tabular}

Fuente: elaboración propia

\section{Discusión}

El grado de conversión activado con fotopolimerizadores oscila entre $48 \%$ y $70 \%$ [9], [10]. Para restauraciones de resina indirectas, se han utilizado procedimientos de curado adicionales para aumentar el grado de conversión y mejorar las propiedades mecánicas [11]. Con un curado adicional a la polimerización inicial se logra ampliar la vibración de la cadena, incrementando y permitiendo así que los radicales libres y los grupos metacrilato hagan más enlaces covalentes, aumentando el grado de conversión. Se ha demostrado que la dureza de los materiales compuestos se correlaciona directamente con el grado de conversión [12].

Los diferentes tratamientos de pospolimerización aplicados a las resinas compuestas surgieron como un método para mejorar las propiedades físicas y mecánicas, como la resistencia a la flexión [13], el desgaste [14], la dureza [15], el aumento de la fuerza a la tracción [16] y la estabilidad del color, entre otros. Es así como trabajando con resinas compuestas en el laboratorio para la fabricación de restauraciones indirectas, obtenemos mejores resultados en comparación con la técnica directa.

Ernst et al. [17] mostraron que el aumento de la microdureza por microondas aumenta los valores de microdureza obteniendo resultados adversos con técnicas que presentan humedad, compatible con los resultados en este trabajo, junto con los resultados de Wendt [18], en los que el microondas presenta los mejores resultados en diversas técnicas de pospolimerización.
La disminución de las propiedades de algunas resinas en agua tiene correlación directa con la absorción [19] y solubilidad en agua de las matrices (degradación hidrolítica) [20], [21].Sankarapandian et al. [22] investigaron el proceso de absorción de agua, su dureza y módulo de elasticidad de varios sistemas resinosos a base BisgmA y análogos, a los cuales se les sustituyó un grupo fenílico del carbón central de la cadena por fluorine, encontrándose que este reducía la absorción de agua en un $10 \%$; además, se reportó que los polímeros fluorinados eran más estables en contacto con el agua, aunque la dureza disminuía y modificaba de manera considerable el módulo de elasticidad de los composites.

Con el fin de disminuir la absorción de agua, se han propuesto otros sistemas entre los que se incluyen los análogos químicos del uretano dimetacrilato (UDMA), que poseen un grupo fenoximetil en la porción periférica o central como grupo alifático [23], [24], que al ser comparado con los sistemas habituales UDMA, reducen entre un 10-30\% la absorción de agua; sin embargo, se ha reportado como efecto secundario la disminución de la resistencia comprensiva [25].

Lombardo et al. [26] realizaron evaluaciones de resinas con procesos de pospolimerización y encontraron mejores resultados de microdureza con pospolimerización en estufa (seca), compatibles con este trabajo. El proceso de curado posterior aumenta la microdureza, pero la polimerización en la microdureza disminuye el autoclave. Por tanto, las resinas con un gran porcentaje en su composición de BisGma presentarán gran absorción de agua y esto acarreará disminución de la microdureza. 


\section{Conclusión}

El proceso de curado posterior por microondas y autoclave mejora los valores en la microdureza de una resina. Por otra parte, el agua destilada a temperaturas altas disminuye la microdureza de la superficie de las resinas compuestas.

\section{Referencias}

[1] Casselli DS, Faria-e-Silva AL, Casselli H, Martins LR. Marginal Adaptation of Composite Restorations in Class v Subjected to Thermal and Mechanical Cycles. J Appl Oral Sci. 2013;21(1):68-73 .

[2] Dietschi D, Scampa U, Campanile G, Holz J. Marginal Adaptation and Seal of Direct and Indirect Class II Composite Resin Restorations: An in Vitro Evaluation. Quintessence Int. 1995;26(2):127-38.

[3] Nandini S. Indirect Resin Composites. J Conserv Dent. 2010;13(4):184-94.

[4] Ferracane JL, Condon JR. Heat Treatment for PostCuring Composite Materials: Properties and Fractography. Dent Mater J. 1992;8:290-5.

[5] Miranda CB, Pagani C, Bottino MC, Benetti AR. A Comparison of Microhardness of Indirect Composite Restorative Materials. J Appl Oral Sci. 2003; 11:157-61.

[6] Soares CJ, Celiberto L, Dechichi P, Fonseca RB, Martins LRM. Marginal Integrity and Microleakage of Direct and Indirect Inlays Composite - And Stereomicroscopic SEM Evaluation. Braz Res Oral. 2005;19(4):295-301.

[7] Silva GR, Simamoto-Júnior PC, da Mota AS, Soares CJ. Mechanical Properties of Light-Curing Composites Polymerized with Different Laboratory PhotoCuring Units. Dent Mater J. 2007;26:217-23.

[8] Sharafeddin F, Sharifi E. The Effect of Microwave/ Laboratory Light Source Postcuring Technique and Wet-Aging on Microhardness of Composite Resin. Dent Res J (Isfahan). 2013;10(3):370-5.

[9] Emami N, Söderholm KJ. How Light Irradiance and Curing Time Affect Monomer Conversion in Light-Cured Resin Composites. Eur J Oral Sci. 2003;111(6):536-42.

[10] Peutzfeldt A, Asmussen E. The Effect of Posturing on Quantity of Remaining Double Bonds, Mechanical Properties, and in vitro Wear of Two Resin Composites. J Dent. 2000;28(6):447-52

[11] Asmussen E, Peutzfeldt A. Mechanical Properties of Heat Treated Restorative Resins for Use in the Inlay/ Onlay Technique. Scand J Dent Res. 1990;98:564-7.
[12] Rueggeberg FA, Ergle JW, Lockwood PE. Effect of Photoinitiator Level on Properties of a Light-Cured and Post-Cure Heated Model Resin System. Dent Mater J. 1997;13(6):360-4.

[13] Da Fonte Porto Carreiro A, Dos Santos Cruz CA, Vergani CE. Hardness and Compressive Strength of Indirect Composite Resins: Effects of Immersion in Distilled Water. J Oral Rehabil. 2004;31(11):1085-9.

[14] Eldiwany M, Powers JM, George LA. Mechanical Properties of Post-Curing Direct and Composites. Am J Dent. 1993;6:222-4.

[15] Santana IL, Lodovici E, Matos JR, Medeiros IS, Miyazaki CL, Rodrigues-Filho LE. Effect of Experimental Heat Treatment on Mechanical Properties of Resin Composites. Braz Dent J. 2009;20:205-10.

[16] Viljanen EK, Skrifvars M, Vallittu PK. Dendritic Copolymers and Particulate Filler Composites for Dental Applications: Degree of Conversion and Thermal Properties. Dental Materials. 2007;23(11):1420-7.

[17] Ernst CP, Kürschner R, Rippin G, Willershausen B. Stress Reduction in Resin-Based Composites Cured with a Two-Step Light-Curing Unit. Am J Dent. 2000;13(2):69-72.

[18] Wendt SL Jr. The Effect of Heat Used as Secondary Cure Upon the Physical Properties of Three Composite Resins. II. Wear, Hardness, and Color Stability. Quintessence Int. 1987;18:351-6.

[19] Mehl A, Hickel R, Kunzelmann KH. Physical Properties and Gap Formation of Light-Cured Composites with and without 'Softstart Polymerization'. J Dent. 1997; 25:321-30.

[20] Bagis YH, Rueggeberg FA. The Effect of Post-Curing, Residual Unreacted Monomer in Commercial Resin Compound Warming. Dent Mater J. 2000;16:244-7.

[21] Rodríguez G, Douglas R, Pereira S, Natalie A. Evolución y tendencias actuales en resinas compuestas. Acta Odontol Venez. 2008;46(3):381-92.

[22] Sankarapandian M, Xu Q, McGrath JE, Taylor DT, Kalachandra S. Influence of Resin Molecular Structure on the Processability and Physical Behavior of a Dental Composite Matrix. J Adv Mater. 1997;28(1):59-63.

[23] Sideridou I, Tserki V, Papanastasiou G. Effect of Chemical Structure on Degree of Conversion in Light-Cured Dimethacrylate-Based Dental Resins. Biomaterials. 2002;23(8):1819-29.

[24] Khatri H, Stansbury J. Investigation of Alternatives to Urethane Dimethacrylate in Dental Resins. J Dent Research. 1999;78:371.

[25] Imazato J, Russell H. Incorporation of Monomer Antibacterial into Resin Composite. J Dent Research. 1994;73(8):1437-43. 
[26] Lombardo GH, Carvalho CF, Galhano G, Souza RO, Junior LN, Pavanelli CA. Influence of Additional Polymerization in the Microhardness of Direct Composite Resins. Cienc Odontol Bras. 2007;10:10-5.
[27] Ferracane JL, Mitchem JC, Condon JR, Todd R. Wear and Marginal Breakdown of Composites with Various Degrees of Cure. J Dent Res. 1997;76:1508-16. 